\title{
Estudio y diseño de módulo de transmisión autónoma de lectura de contadores de energía eléctrica integrado a loT
}

Javier Rojas-Cárdenas ${ }^{1}$

Corporación Universitaria Unitec

67151500@unitec.edu.co

Yeison Estiven Otavo-Vargas ${ }^{2}$

Corporación Universitaria Unitec

67151502@unitec.edu.co

Juan Felipe Gutiérrez ${ }^{3}$

Corporación Universitaria Unitec

juan.gutierrez@unitec.edu.co

DOI: https://doi.org/10.21158/23823399.v8.n0.2020.2735

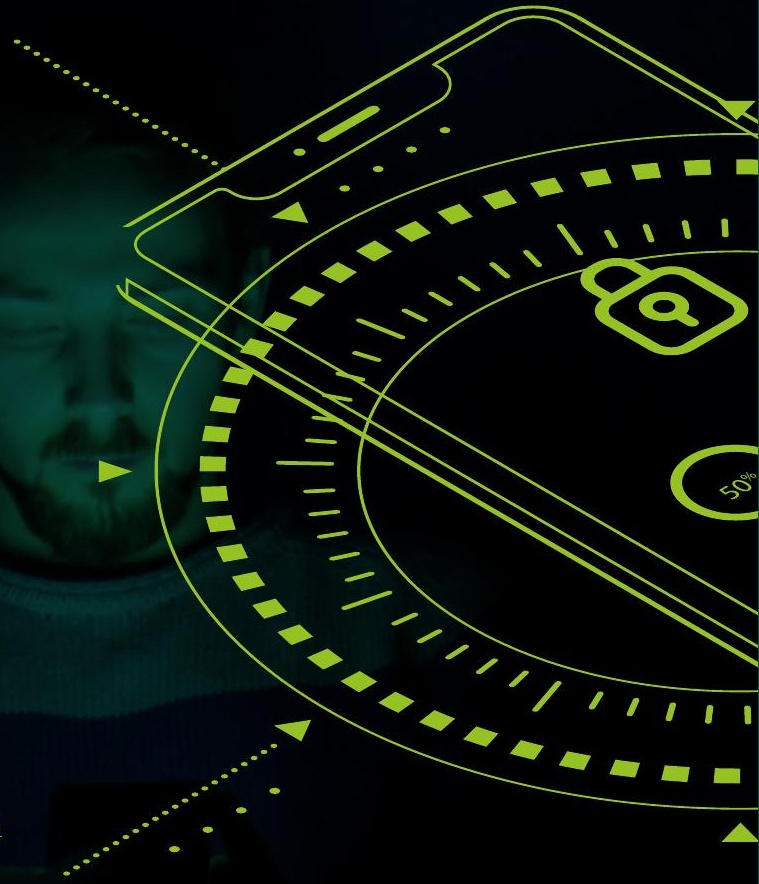

Fecha de recepción: 12 de marzo de 2020

Fecha de aprobación: 10 de junio de 2020

Cómo citar este artículo: Rojas-Cárdenas, J.; Otavo-Vargas, Y. E.; Gutiérrez, J.F. (2020). Estudio y diseño de módulo de transmisión autónoma de lectura de contadores de energía eléctrica integrado a loT.

Revista Ontare, 8, 125-149. DOI: https://doi.org/10.21158/23823399.v8.n0.2020.2735

\footnotetext{
${ }^{1}$ Ingeniero en Telecomunicaciones - Corporación Universitaria Unitec. ORCID: https://orcid.org/0000-0001-9967-271X ${ }^{2}$ Ingeniero en Telecomunicaciones - Corporación Universitaria Unitec. ORCID: https://orcid.org/0000-0002-5171-9758

${ }^{3}$ Ingeniero electrónico de la Universidad Nacional de Colombia. Magister en Telecomunicaciones, de la Universidad Nacional de Colombia. ORCID: https://orcid.org/0000-0001-8509-8075
} 



\section{RESUMEN}

El proyecto tuvo como finalidad diseñar e implementar un módulo encargado de tomar lecturas de consumo de energía eléctrica, las cuales se transmiten por medio de las tecnologías móviles de acceso a internet actuales - GSM e loT- a un servidor web en el que las puede consultar el cliente haciendo uso de un login de ingreso a las lecturas - usuario y contraseña-. Su funcionamiento se basa el uso de una tarjeta microcontroladora unificada a unos dispositivos de medición de tensión y amperaje previamente calibrados, de modo que realiza una medición comparativa de estos mismos valores en la red eléctrica en actual operación. Finalmente, los datos se envían al servidor alojado en la web mediante un módulo de conexión GSM asociado a una tarjeta SIM previamente registrada con cualquiera de los operadores móviles en operación en el país. En este artículo se explican el diseño y el funcionamiento de un módulo que tiene como objetivo principal tomar el valor fiel de una medición de potencia consumida. La transmisión de datos desde el módulo de transmisión de datos al servidor dedicado se generó de manera correcta y continua en las múltiples pruebas realizadas durante un periodo de tiempo prolongado. Así mismo, la tecnología implementada para la transmisión de datos - GSM- demostró ser la elección más factible en términos de infraestructura y alcance en la implementación del proyecto debido a la gran acogida que tiene la tecnología utilizada en el país, puesto que ahorra costes en la implementación de una red de comunicación.

Palabras clave: consumo de energía eléctrica; medición de tensión; medición de amperaje; transmisión autónoma de datos; tecnologías móviles de acceso a internet; lectura contadores energía eléctrica. 


\section{Study and design of an autonomous transmission module for reading electrical energy consumption meters integrated to IOT}

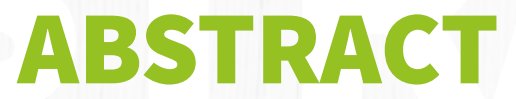

This project aimed at designing and implementing a module for taking readings of electrical energy consumption, which at this moment are being transmitted to a web server by using the current mobile internet access technologies -GSM and IoT- allowing the client to have access to his information with a login to enter the readings -user name and password-. Its operation is based on the use of a microcontroller card that has been unified to some previously calibrated voltage and amperage measurement devices, so that it makes a comparative measurement of these values in the ongoing operation. Finally, the data is sent to the server that is hosted on the web by means of a GSM connection module associated to a SIM card that has been registered previously with any of the mobile service providers in operation in the country. This article explains the design and operation of a module whose main objective is to take the true value of a power consumption measurement. The transmission of the data from the data transmission module to the dedicated server was correctly and continuously generated in the multiple tests performed over a long period of time. Likewise, the technology that was implemented for the transmission of the data -GSMproved to be the most feasible choice in terms of infrastructure and scope in the implementation of the project, due to the great acceptance that this technology has in the country, since it saves costs in the implementation of a communication network.

Keywords: electric energy consumption; voltage measurement; amperage measurement; autonomous transmission of data; mobile technologies for internet access; reading of electrical consumption meter. 


\section{Estudo e desenho de módulo de transmissão autônoma de leitura de contadores de energia elétrica integrado a loT}

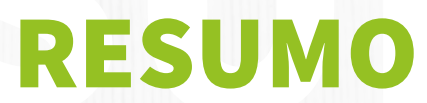

O projeto teve como finalidade desenhar e implementar um módulo encarregado de tomar leituras de consumo de energia elétrica, as quais se transmitem por meio das tecnologias móveis atuais de acesso à internet GSM e IOT - a um servidor web que pode ser consultado pelo cliente fazendo uso de um login de entrada às leituras - usuário e senha. Seu funcionamento se apoia no uso de um cartão microcontrolador unificado a uns dispositivos de medida de tensão e amperagem previamente calibrados, de maneira que realiza uma medida comparativa destes mesmos valores na rede elétrica em atual operação. Finalmente, os dados enviam-se ao servidor alojado no site mediante um módulo de conexão GSM associado a um cartão SIM previamente registrado com qualquer dos operadores móveis em operação no país. Neste artigo explicam-se o desenho e o funcionamento de um módulo que tem como objetivo principal tomar o valor fiel de uma medida de potência consumida. A transmissão de dados desde o módulo de transmissão de dados ao servidor dedicado gerou-se de maneira correta e contínua nas múltiplas provas realizadas durante um período de tempo prolongado. A tecnologia implementada para a transmissão de dados - GSM - demonstrou ser a escolha mais factível em termos de infraestrutura e alcance na implementação do projeto devido à grande acolhida que tem a tecnologia utilizada no país, já que poupa custos na implementação de uma rede de comunicação.

Palavras-chave: consumo de energia elétrica; medida de tensão; medida de amperagem; transmissão autônoma de dados; tecnologias móveis de acesso à internet; leitura contadores energia elétrica. 


\section{Etude et conception d'un module de transmission autonome pour la lecture de compteurs électriques intégrés à l'IoT}

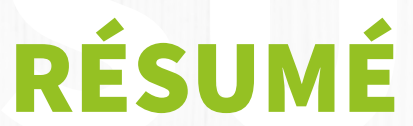

Ce projet a comme objectif la conception et la mise en place d'un module chargé de mesurer les consommations d'énergie électrique transmises via l'internet mobile-GSM et IoT - à un serveur où elles pourront être consultées par le client grâce à identifiant utilisateur et un mot de passe. Le fonctionnement du module repose sur l'utilisation d'une carte de microprocesseur connectée à des dispositifs de mesure de tension et d'intensité préalablement calibrés, de sorte que le module réalise une mesure comparative desdites valeurs sur le réseau électrique national. Enfin, les données sont envoyées au serveur hébergé sur internet via un module de connexion GSM associé à une carte SIM préalablement enregistrée auprès de l'un des opérateurs mobiles présent localement. Cet article explique la conception et le fonctionnement d'un module dont l'objectif est de prendre la valeur exacte d'une mesure de puissance consommée. La transmission des données du module vers le serveur dédié a fonctionné correctement et en continu lors des multiples tests effectués sur une période de temps long. De même, la technologie mise en œuvre pour la transmission de données - GSM - s'est avérée être le meilleur choix en termes d'infrastructure et de portée lors de la mise en œuvre du projet en raison de l'utilisation répandue de cette technologie permettant des économies de coûts évitant la construction d'un autre réseau de communication.

Mots clés: consommation d'énergie électrique; mesure de tension; mesure de l'ampérage; transmission de données autonome; technologies d'accès à internet mobile; lecture de compteur d'énergie électrique. 


\section{Introducción}

En este artículo se explican el diseño y el funcionamiento de un módulo que tiene como objetivo principal tomar el valor fiel de una medición de potencia consumida, mediante las tecnologías de trasmisión de datos móviles almacenados en una base de datos - alojada en un servidor remoto- y que permiten al usuario final consultar el consumo por medio de una plataforma web a la que ingresa con un inicio de sesión único. Para esto requiere tener conocimiento de conceptos técnicos, los cuales se describen a continuación.

\subsection{Corriente alterna (AC)}

Una corriente que cambie de sentido a intervalos de tiempo recibe el nombre de corriente alterna. La corriente que tenemos en las bases de enchufe de casa se denomina corriente alterna senoidal.

La forma de la onda senoidal es periódica, ya que se reproduce de forma idéntica en intervalos de tiempo iguales (Garrigós, 2011).

\section{Figura 1. Gráfica de corriente alterna}

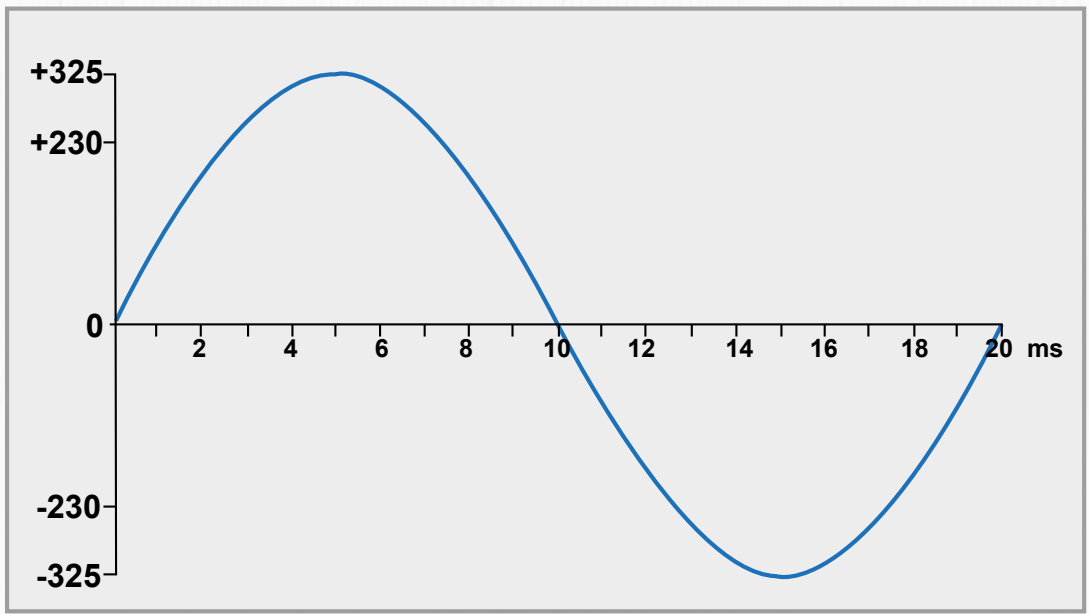

Fuente. Elaboración propia. 


\subsection{Sensor de corriente}

La familia SCT-013 son sensores de corrientes no invasivos que permiten medir la intensidad que atraviesa un conductor sin necesidad de cortar o modificar el conductor. Es posible emplear estos sensores con un procesador como Arduino para medir la intensidad o potencia consumida por una carga.

Los sensores SCT-013 son transformadores de corriente, dispositivos de instrumentación que proporcionan una medición proporcional a la intensidad que atraviesa un circuito. La medición se realiza por inducción electromagnética.

Los sensores SCT-013 disponen de un núcleo ferromagnético partido como una pinza- que permite abrirlo a fin de arrollar un conductor de una instalación eléctrica sin necesidad de cortarlo.

La precisión del sensor puede ser de 1-2 \%, pero para esto es muy importante que el núcleo ferromagnético se cierre de forma adecuada. Hasta un pequeño hueco de aire puede introducir desviaciones del $10 \%$ (Llamas, 24 enero de 2017).

\subsection{Sensor de voltaje}

El módulo transformador de voltaje alterno ZMPT101B permite medir voltaje alterno como el que se encuentra en la mayoría de hogares. Este voltaje AC no puede medirse de forma directa por el ADC del Arduino, pues escapa al rango de entrada $-0 \mathrm{~V}$ a $5 \mathrm{~V}$-. El módulo ZMPT101B soluciona el problema al reducir el voltaje $A C$ de entrada a un voltaje menor que pueda leer el Arduino o cualquier otro microcontrolador.

El módulo lo integra un transformador que cumple la función de aislamiento galvánico para mayor seguridad en el uso. El lado primario del transformador se conecta al voltaje alterno que se desea medir. En el lado secundario del transformador se encuentra un divisor de tensión y un circuito con amplificador operacional -OPAMP LM358 - para adicionar un desplazamiento - offset-a la salida análoga. 
Así mismo, soporta voltajes de entrada de hasta 250 VAC y entrega una onda senoidal de amplitud regulable por un potenciómetro en placa. La onda senoidal de salida se encuentra desplazada positivamente con el propósito de que esta no tenga voltajes negativos y así estar en capacidad de leerla completamente con el ADC. El desplazamiento depende del voltaje con el que se alimente el módulo: si el voltaje de alimentación es de $5 \mathrm{~V}$ el desplazamiento será de 2,5 V, y si se alimenta el módulo con 3,3 V el desplazamiento será de 1,65 V. El circuito de acondicionamiento de señal permite que el voltaje de salida del módulo pueda leerlo cualquier microcontrolador con entrada analógica $-A D C$ - . De esta forma es posible leer el voltaje instantáneo y realizar cálculos de energía, como, por ejemplo, voltaje pico a pico -Vpp-y voltaje eficaz - Vrms - (Naylamp Mechatronics, s. f.).

\subsection{El Arduino Leonardo}

El Arduino Leonardo es una placa de microcontrolador basada en el ATmega32u4 - hoja de datos-. Tiene 20 pines digitales de entrada/salida -de los cuales siete pueden usarse como salidas PWM y 12 como entradas analógicas-, un cristal de $16 \mathrm{MHz}$ oscilador, una conexión micro USB, un conector de alimentación, un encabezado ICSP y un botón de reinicio. Contiene todo lo necesario para soportar el microcontrolador; simplemente se debe conectar a una computadora con un cable USB o encenderlo con un adaptador de CA a CC o batería para comenzar.

Leonardo difiere de todas las placas anteriores en las que el ATmega32u4 tiene comunicación USB incorporada, de modo que elimina así la necesidad de un procesador secundario. Esto permite que el Arduino aparezca aun conectado a una computadora como un ratón y teclado, además de un puerto serie/COM virtual - CDC - (Arduino, s. f.). 


\subsection{MySQL}

Es un sistema de administración de bases de datos relacionales rápido, robusto y fácil de usar. Se adapta bien a la administración de datos en un entorno de red, especialmente en arquitecturas cliente/servidor. Se proporciona con muchas herramientas y es compatible con muchos lenguajes de programación. Es el más célebre del mundo open source, en particular gracias a su compatibilidad con el servidor de páginas web Apache y el lenguaje de páginas web dinámicas PHP.

MySQL se difunde como open source. Así, cualquiera que utilice MySQL tiene acceso al código fuente y puede modificar el código con el fin de adaptarlo a sus propias necesidades.

Debido a su licencia GNU General Public Licence - GLP-, toda persona puede descargar, instalar y utilizar MySQL libremente sin ninguna aportación económica (Thibaud, 2006).

\subsection{Módulo Sim800L}

El módulo permite la transmisión de datos voz y texto haciendo uso de los servicios de la tecnología GPRS; requiere de un microcontrolador para realizar control sobre este a través de comunicación serial.

Su ficha técnica es la siguiente:

- voltaje de operación: 3,4 V-4,4V DC;

- nivel lógico de $3 \mathrm{~V}$ a $5 \mathrm{~V}$;

- consumo de corriente -máx-: 500 mA;

- consumo de corriente -modo de reposo-:0,7 mA;

- interfaz: serial UART;

- quad-band $850 / 900 / 1800 / 1900 \mathrm{MHz}$ - se conectan a cualquier red mundial GSM con cualquier SIM 2G-;

- trabaja solo con tecnología 2G;

- hacer y recibir llamadas de voz usando un auricular o un altavoz de $8 \Omega$ externo + micrófono electret;

- enviar y recibir mensajes SMS; 
- enviar y recibir datos GPRS - TCP/IP, HTTP, etc.--;

- escanear y recibir emisiones de radio FM;

- controlado por comandos AT;

- interfaz de comandos AT con detección «automática» de velocidad de transmisión;

- soporta A-GPS;

- datos GPRS (Electropro, s. f.).

\section{Implementación}

Figura 2. Diagrama de implementación y diseño del prototipo

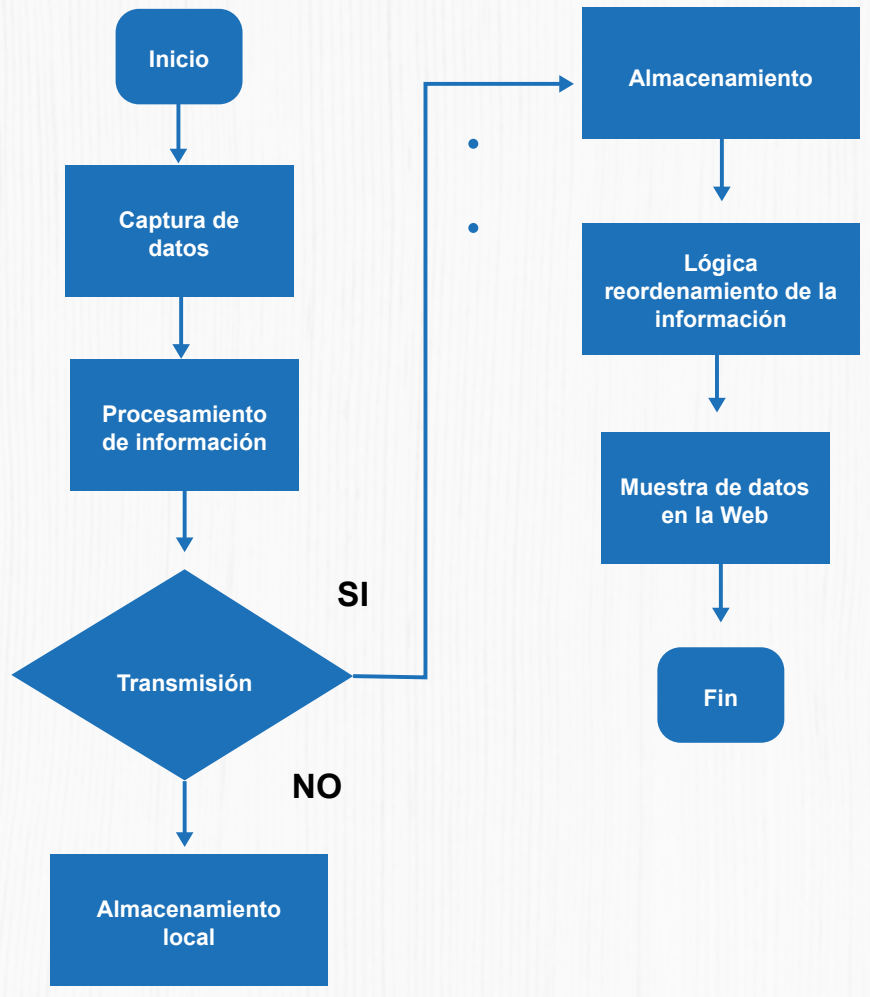

Fuente. Elaboración propia. 


\subsection{Estructura del código principal}

En el interior de la estructura principal ejecutada dentro de la función «Void Setup ()» « «Void Loop ()» se realizó el formato de comunicación con el módulo sim800 y la ejecución de la función «comandosAT ()», encargada del encapsulamiento de los datos censados para ser enviados a través de la red GSM.

La comunicación con el módulo Sim se realiza mediante la activación de una serie de tareas que, en conjunto, permiten activar el módulo, enlazarlo a la red móvil y prepararlo para activar el envió de datos a través de la red móvil del operador seleccionado.

\section{Figura 3. Línea de comandos para activación del módulo SIM}

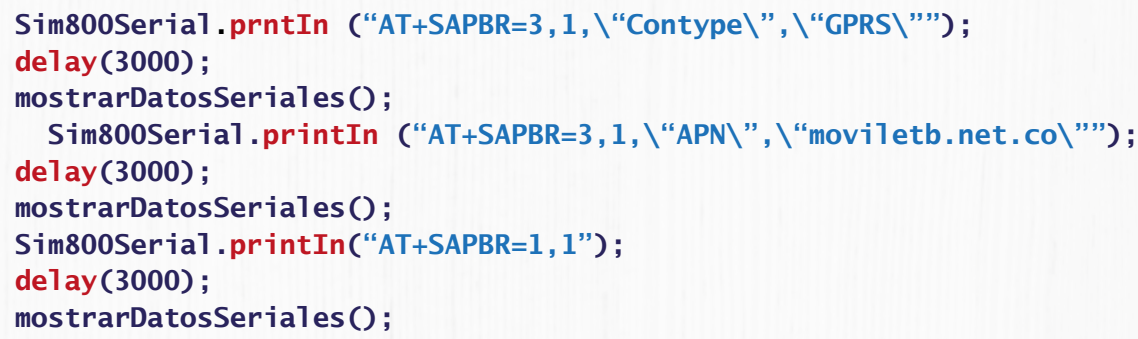

Fuente. Elaboración propia.

\subsubsection{Función «comandosAT()».}

El uso de la función permitió generar una estructura capaz de enlazar los datos de medición obtenidos con los dispositivos de lectura al módulo Sim, el cual se encarga de enviarlos vía internet al servidor por medio de la red del operador móvil. 


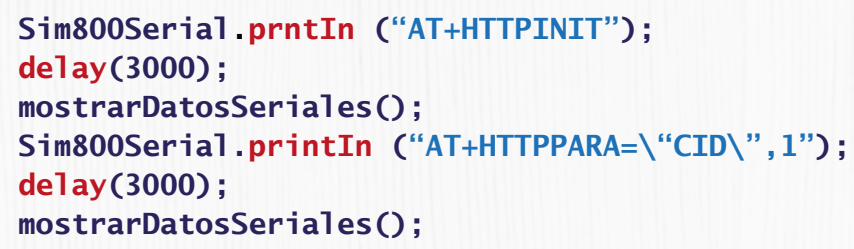

\section{Fuente. Elaboración propia.}

Como se evidencia en la figura 4, el proceso de transmisión de los datos capturados se genera mediante una conexión a través del puerto HTTP - 80-, con lo cual se generó la apertura de la conexión y se debe obtener una respuesta afirmativa de conexión por medio del monitor serial.

\subsubsection{Arreglo del encapsulamiento de los datos a transmitir.}

Una de la tareas que se ejecutaron dentro de la función «comandosAT()» es el esquema para el envió de los datos, el cual se planteó tal como se muestra en la figura 5.

\section{Figura 5. URL Trasmisión datos al servidor}

Sim800Serial . prntIn ("AT+HTTPpara=\"URL\", \“www . modulomedicion . on 1 ine/1ecturas . php?pass=12345);

Fuente. Elaboración propia.

El primer paso de la línea de programación en la que se encapsulan los datos es determinar la conexión con la URL de acceso al servidor. Para esto se declaró el nombre de la página web, así como se informó el nombre de la base de datos a consultar - «lecturas.php»-y la contraseña de acceso a esta base. 


\section{\&id_sensor $=34 \mathrm{AB}$}

Fuente. Elaboración propia.

El siguiente paso fue el ingreso de las variables consultables dentro de la base de datos, comenzando por la variable encargada de asignar una identificación al módulo desde el que se están enviando los datos.

Figura 7. Variables corriente, voltaje y potencia

\&corriente="+ String(Irms) + "\&vo1taje=" + String(voltaje) + "\&potencia=" + String(potencia) + "\"”);

Fuente. Elaboración propia.

Por último, se ingresaron los datos de las variables en las que se almacenaron los datos a transmitir referentes a corriente, voltaje y potencia. Estos valores se transformaron a un formato de texto para que fuera posible manipularlos en el servidor.

\subsection{Configuración de almacenamiento de la información recibida del microcontrolador}

Se realizó la elaboración de las tablas que componen la base de datos, esta también importante para los datos que se reciben del sensor. La conformación de las tablas se diseñó de la siguiente forma. La primera, llamada Usuarios, en la que se almacenan todos los datos de las personales y los datos del predio de las personas que se inscriban por la página web para la obtención del servicio; luego, una segunda tabla que contiene todos los datos del sensor - marca serial-y almacena los datos que recibe el sensor -corriente, voltaje y potencia-. 
Figura 8. Tablas de la base de datos

vo u842804775 dalus lecturas
a id : int(255)
ø fecha : timestamp
(i) id sensor: Iongtext
\# corricnto: float
\# voltaje: float
\# potencia : float

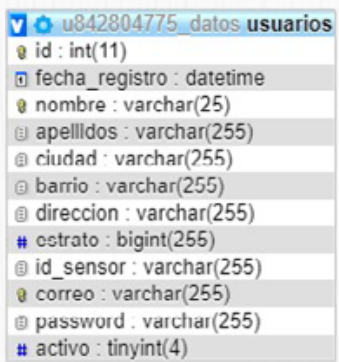

Fuente. Elaboración propia.

A nivel de servidor se realizó la copia del código que previamente se diseñó en el servidor de pruebas y se validó la funcionalidad de los servicios elaborados, teniendo en cuenta los cambios en algunos archivos que contenían direcciones ip a nivel local, de manera que se actualizaron al nuevo dominio adquirido. En la imagen de la figura 9 se puede apreciar uno de los archivos más importantes a nivel de conexión, ya que es aquí que indicamos la conectividad a la base de datos y, de esta forma, se logra que los datos recibidos se almacenen de forma correcta.

\section{Figura 9. Configuración archivo de conexión a bd}

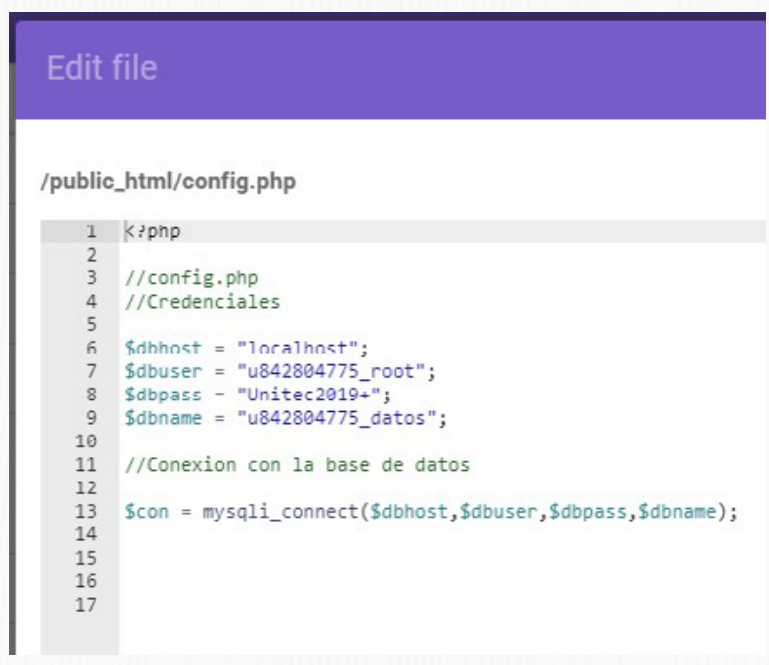

Fuente. Elaboración propia. 
Este archivo config.php se compone de la siguiente estructura:

- nombre del servidor;

- usuario de ingreso al servidor;

- contraseña de ingreso al servidor;

- nombre de la base de datos.

Otro de los archivos importantes en la recepción de los datos es lecturas. php; su importancia radica en que es a este archivo al que se apunta de forma específica desde el Arduino para el almacenamiento de los datos. Se compone de los parámetros que se describen a continuación.

Inicialmente, se tiene la conexión con el archivo config.php explicado, con el fin de obtener la conexión a la base de datos; en el «if» se configura una seguridad, es decir, solo quien tenga la contraseña puede enviar a esa URL.

Luego, se configuran las variables llamando la conexión a la base de datos y utilizando los métodos GET a fin de obtener cada una de las variables que vienen desde el Arduino -id_sensor, corriente, voltaje, potencia-.

Enseguida se le ordena al sistema, por medio de una sentencia MySQL, que inserte cada uno de los valores almacenados en las variables en la tabla lecturas que se configuraron previamente en la base de datos.

Figura 10. Código obtención de variables

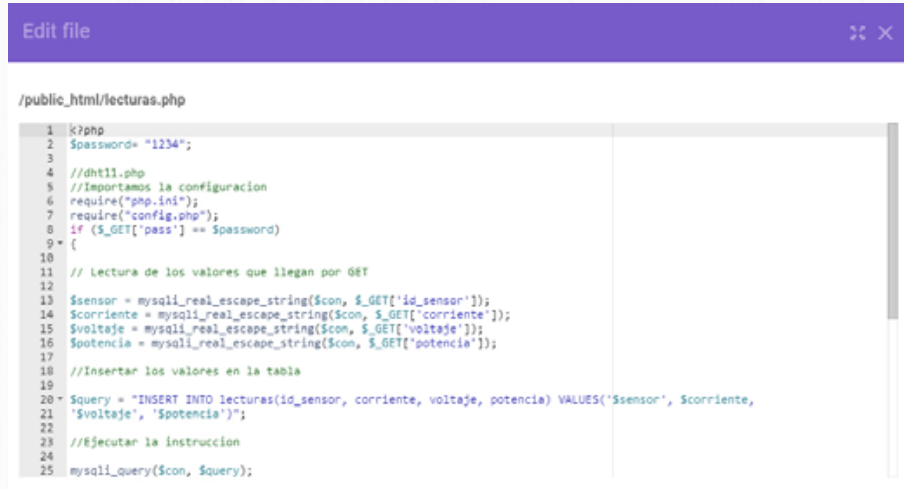


Finalmente, la última línea de código le ordena al sistema que se ejecuten las instrucciones dadas. Es de esta forma que se almacenan los datos enviados desde el microcontrolador en la base. En la imagen de la figura 10 se evidencia una muestra de algunas lecturas que se tomaron durante el funcionamiento del módulo y se fueron almacenando según la instrucción dada.

Dentro de la creación de la página se creó un entorno que permite al usuario que quiera usar el servicio completar un corto formulario con información básica, con el fin de que se pueda visualizar la información de una forma coherente y clara, tal como se muestra en la imagen de la figura 11.

Figura 11. Interfaz de acceso

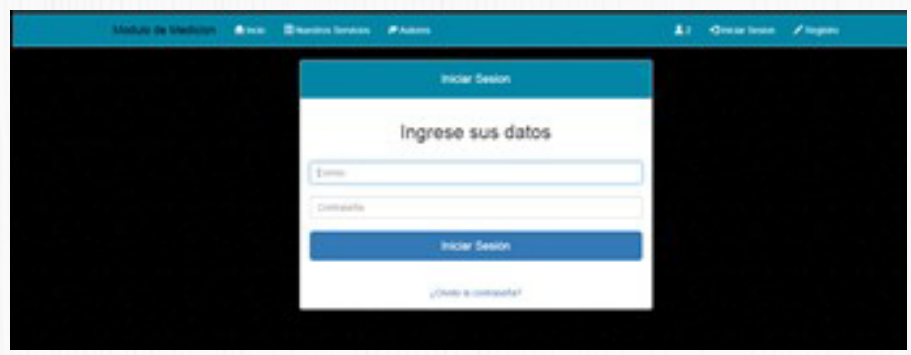

Fuente. Elaboración propia.

\subsubsection{Gráfica en barras.}

Se decidió que en esta misma plantilla «contador.php» se realizara la visualización de la información recibida por gráficas, para que el usuario obtenga de una forma más entendible la información que recibe de los sensores. Para el diseño a nivel de programación de este tipo de gráficas se usa la librería llamada plotly, una librería de código abierto obtenida de la página web https://plot.ly/r/, en la cual se realizó la configuración con la consulta para que se obtuvieran los datos de los consumos por meses. 


\section{LECTURA DEL MEDIDOR}

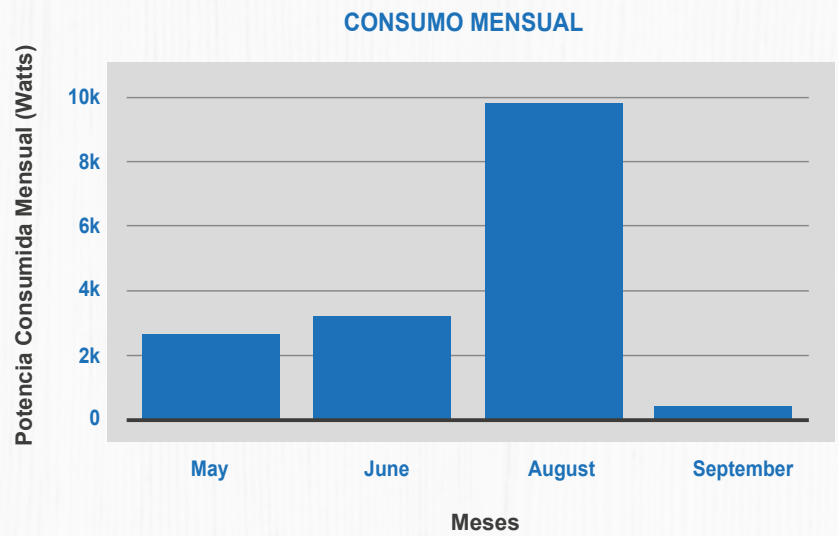

Fuente. Elaboración propia.

En esta gráfica el usuario tiene la facilidad de evidenciar el consumo de energía en el mes actual y, adicionalmente, cuenta con los datos históricos de los meses anteriores. De esta forma se pueda tener un control y así el usuario está en capacidad de determinar en qué periodos las lecturas han tenido un mayor consumo, lo cual permite regular el consumo de energía.

\subsection{Gráfica de consumo en tiempo real}

Tanto la fecha como la potencia toman los valores de los ejes $x y$, los cuales se adjuntan al código de la gráfica y, finalmente, nos da como resultado el muestreo de los datos que llegan del microprocesador, como se expone en la figura 13. 


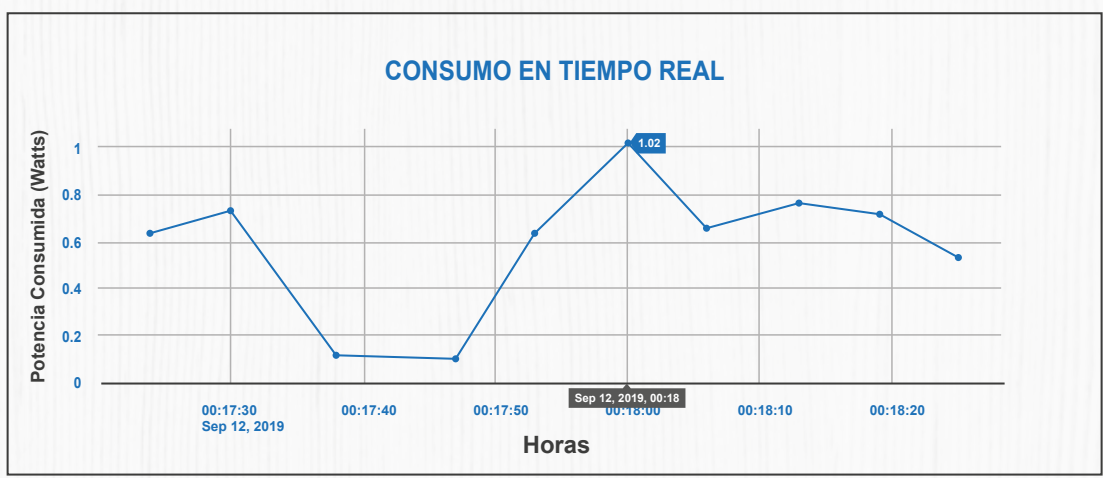

Fuente. Elaboración propia.

Esta gráfica le será muy útil al usuario ya que se puede apreciar que el módulo está en funcionamiento tomando las lecturas y también muestra en qué momentos ocurren picos de potencia cuando se genera un consumo fuera de lo normal.

\section{Resultados}

\subsection{Proceso de calibración del sensor de corriente SCT-013}

A fin de conseguir un valor de calibración acorde a las lecturas de amperaje suministradas por la acometida eléctrica diseñada, se deben conocer las características técnicas del sensor y cómo se genera la toma de las lecturas.

El sensor SCT-013 maneja una toma de lecturas reconocidas a través de los pines analógicos de la tarjeta microcontroladora. El sensor refleja lecturas que se reciben desde un rango de 0 a 1024, siendo el valor máximo correspondiente con el voltaje de entrada máximo del microcontrolador que es de $5 \mathrm{~V}$. 
La lectura de un amperaje alterno para obtener un valor de corriente eficaz (RMS) requiere generar un muestreo de valores tanto positivos como negativos; la tarjeta microcontroladora solo tiene la capacidad de recibir valores positivos, motivo por el cual las lecturas que tomen los valores negativos de la señal serán siempre tomadas como cero.

La solución implementada consistió en diseñar un circuito off set que permitió generar el corrimiento de la señal recibido con la finalidad de que pueda ser medida dentro del rango de voltaje de entrada máximo permitido $-0-5 \mathrm{~V}-$.

\section{Figura 14. Circuito off set implementado}

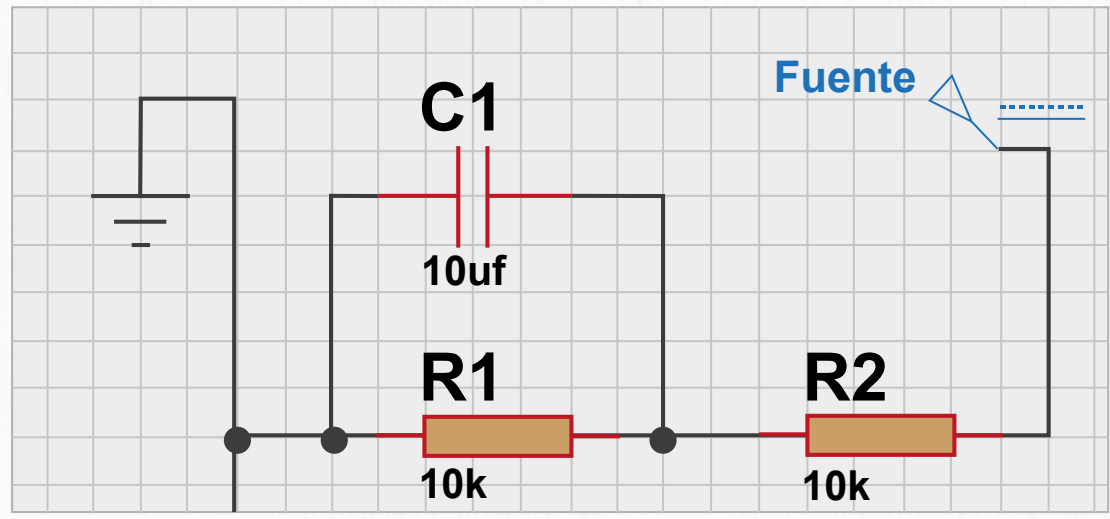

Fuente. Elaboración propia.

Por ende, luego de diseñar el circuito off set fue necesario generar un código de programación que permitió tomar las lecturas del pin analógico y que se visualizaran por medio del monitor serie.

Figura 15. Línea de código para la lectura de valores

\section{Sensor=analogRead(A0); Serial.printIn (sensor);}

Fuente. Elaboración propia. 
La aplicación de las líneas que se muestran en la figura 16 permitió capturar los valores del sensor y luego imprimirlos en el monitor serial de la aplicación Arduino.

Figura 16. Señal reflejada en el monitor serie

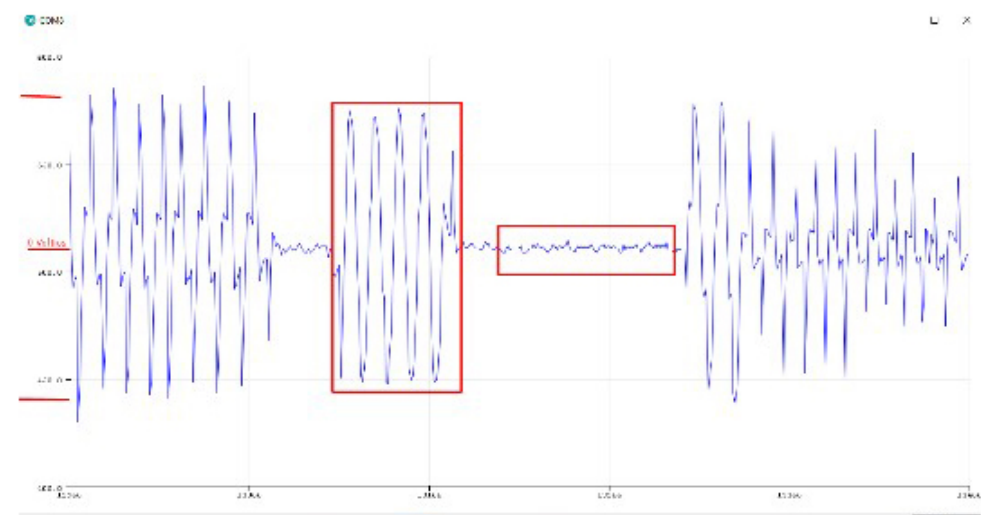

Fuente. Elaboración propia.

\subsection{Calibración del sensor de voltaje ZMPT101B}

La ventaja del sensor de voltaje, que se implementó por sobre el sensor de amperaje, fue que las especificaciones técnicas de la datasheet permitieron aclarar que las lecturas tomadas por este hicieron posible identificar cómo en la entrada analógica de la placa los valores de voltaje del sensor, que van de los 0 voltios a máximo 250 voltios, podían interpretarse en la entrada analógica como un valor de 512 para una lectura de 0 voltios, y de 1024 para un valor máximo de 250 voltios. Por esta razón no fue necesario implementar un circuito offset.

Figura 17. Calibración del sensor de voltaje ZMPT101B

\section{Sensor = analogRead(A1) \\ Serial.printIn (sensor);}

Fuente. Elaboración propia. 


\subsection{Cálculo del factor de potencia aparente}

Luego de calibrar los sensores para obtener los valores de corriente y voltaje, respectivamente, se procede con la obtención de la potencia partiendo de multiplicar el valor de voltaje obtenido con el valor de corriente a partir de los valores RMS de estos.

Figura 18. Cálculo de potencia aparente -S-

$$
S=U \cdot I
$$

Fuente. Elaboración propia.

\subsection{Transmisión de los datos obtenidos a través de la red GSM}

Se realizó una serie de pruebas mediante las cuales fue posible determinar la recepción de la información enviada. Durante el proceso de construcción del servidor de alojamiento de los datos fue necesario generar unas pruebas de envío que pudieran comprobar el funcionamiento del módulo.

La primera prueba consistió en realizar una captura de datos y transmitirlos a un servidor de alojamiento de datos gratuito bajo el dominio https:// thingspeak.com/; esto permitió corroborar el funcionamiento correcto del módulo.

Figura 19. Datos subidos a www.thingspeak.com
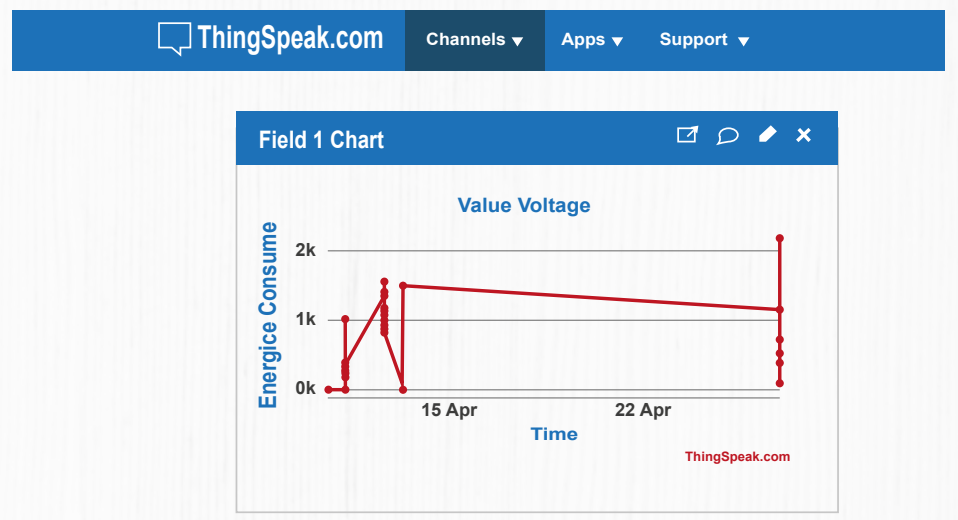
Luego de comprobar el funcionamiento del módulo se opta por hacer uso de código para el encapsulamiento y el envió de los datos al servidor diseñado.

Figura 20. Transmisión de los datos reflejado en monitor serial de Arduino

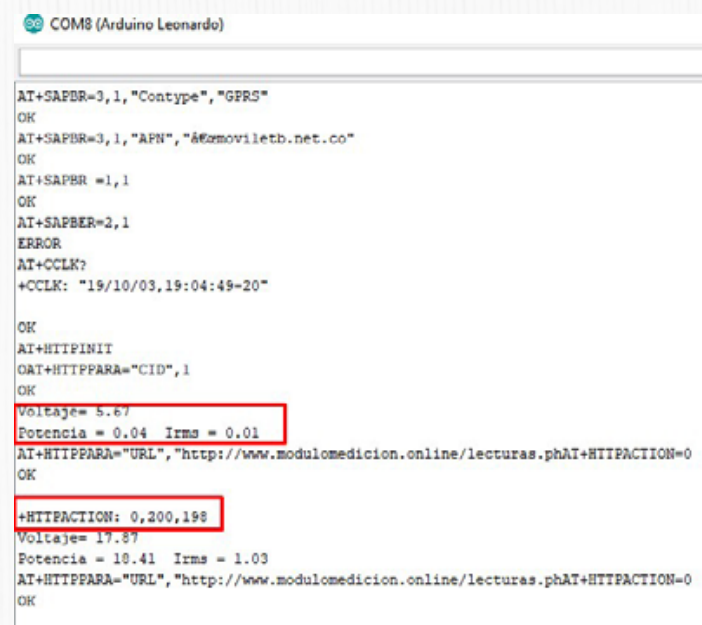

Fuente. Elaboración propia.

Los datos transmitidos en la figura 20 se reciben en el servidor. Luego, se muestran en una gráfica diseñada con la finalidad de ser actualizados de manera periódica, para ser transmitidos en tiempo real.

Figura 21. Gráfica de consumo en tiempo real

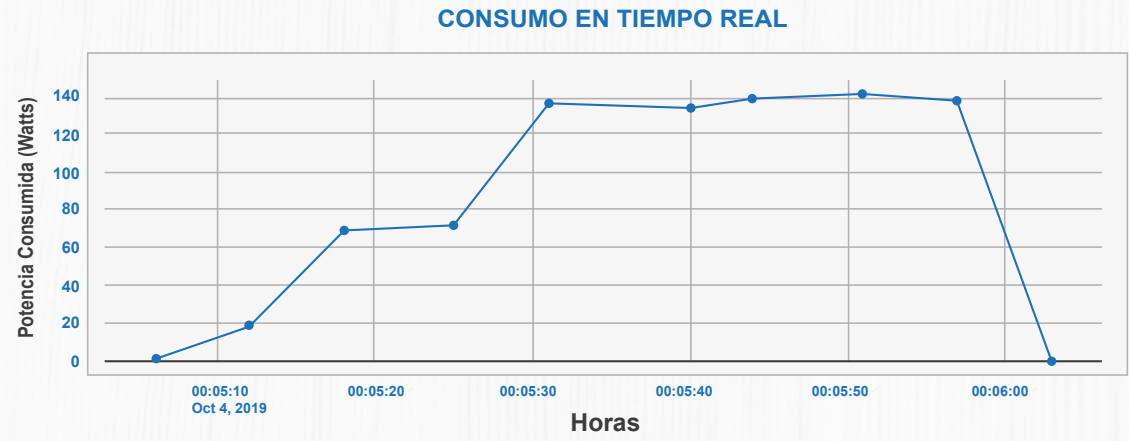

Fuente. Elaboración propia. 


\section{Conclusiones}

La transmisión de datos desde el módulo de transmisión de datos al servidor dedicado se generó de manera correcta y continua en las múltiples pruebas realizadas durante un periodo de tiempo prolongado. El servidor fue capaz de mostrar los datos recibidos en modelos gráficos e históricos de consumo que fueron acordes con el tiempo y el momento en el que se transmitieron por el módulo. Como método de contingencia, en caso de tener problemas de cobertura para la transmisión de los datos, se debe implementar un mecanismo de almacenamiento local de las lecturas que se toman con la finalidad de reenviarlas en cuanto se tenga conexión.

El proceso de calibración de los sensores es acorde con las lecturas residenciales previamente tomadas del enchufe eléctrico mediante el uso de un multímetro, lo que garantiza la fiabilidad de la medición. Así mismo, la tecnología implementada para la transmisión de datos - GSM- es la elección más factible en términos de infraestructura y alcance en la implementación del proyecto debido a la gran acogida que tiene la tecnología utilizada en el país, ya que ahorra costes en implementación de una red de comunicación. 


\section{Referencias}

Arduino. (s. f.). Arduino Leonardo. Recuperado de https://bit.ly/39zjy9T

Electropro. (s. f.). Módulo GSM Sim800. Recuperado de https://bit.ly/2Vtqm0A

Garrigós, J. (2011). Ciclo Formativo de Grado Superior Técnico Superior en Industria Alimentaria. Introducción a la Electricidad. I. E. S. Andrés de Vandelvira. Castilla- La Mancha, España.

Llamas, L. (24 enero de 2017). Sensor de corriente eléctrica no invasivo con arduino y Sct-013. Luisllamas.es. Recuperado de https://bit.ly/2JjblGT

Naylamp Mechatronics. (s. f.). Transformador de voltaje AC-ZMPT101B. Recuperado de https://bit.ly/3otfkFd

Thibaud, C. (2006). Recursos informáticos MySQL 5. Barcelona: Ediciones ENI. 